\title{
Generating a Lexicon Without a Language Model: Do Words for Number Count?
}

\section{Citation}

Spaepen, Elizabet, Marie Coppola, Molly Flaherty, Elizabeth Spelke, and Susan Goldin-Meadow. 2013. Generating a Lexicon Without a Language Model: Do Words for Number Count? Journal of Memory and Language 69, no. 4: 496-505.

\section{Published Version}

doi:10.1016/j.jml.2013.05.004

\section{Permanent link}

http://nrs.harvard.edu/urn-3:HUL.InstRepos:13406009

\section{Terms of Use}

This article was downloaded from Harvard University's DASH repository, and is made available under the terms and conditions applicable to Other Posted Material, as set forth at http:// nrs.harvard.edu/urn-3:HUL.InstRepos:dash.current.terms-of-use\#LAA

\section{Share Your Story}

The Harvard community has made this article openly available.

Please share how this access benefits you. Submit a story.

Accessibility 


\title{
Generating a lexicon without a language model: Do words for number count?
}

\author{
Elizabet Spaepen ${ }^{a}$, Marie Coppola $^{\mathrm{a}, \mathrm{c}}$, Molly Flaherty ${ }^{\mathrm{a}}$, Elizabeth Spelke ${ }^{\mathrm{b}}$, and Susan Goldin- \\ Meadow $^{\mathrm{a}}$ \\ aUniversity of Chicago, Department of Psychology, 5848 S. University Ave., Chicago IL 60637, \\ liesje@uchicago.edu, mflaherty@uchicago.edu, sgm@uchicago.edu \\ bHarvard University, Department of Psychology, William James Hall, 33 Kirkland Ave., \\ Cambridge, MA 02138, spelke@wjh.harvard.edu
}

\begin{abstract}
Homesigns are communication systems created by deaf individuals without access to conventional linguistic input. To investigate how homesign gestures for number function in short-term memory compared to homesign gestures for objects, actions, or attributes, we conducted memory span tasks with adult homesigners in Nicaragua, and with comparison groups of unschooled hearing Spanish speakers and deaf Nicaraguan Sign Language signers. There was no difference between groups in recall of gestures or words for objects, actions or attributes; homesign gestures therefore can function as word units in short-term memory. However, homesigners showed poorer recall of numbers than the other groups. Unlike the other groups, increasing the numerical value of the tobe-remembered quantities negatively affected recall in homesigners, but not controls. When developed without linguistic input, gestures for number do not seem to function as summaries of the cardinal values of the sets (four), but rather as indexes of items within a set (one-one-one-one).
\end{abstract}

\section{Keywords}

numerical cognition; digit span; short-term memory; Nicaraguan Sign Language; homesign; lexical representation; gesture

\begin{abstract}
Deaf individuals whose hearing losses prevent them from learning the spoken language that surrounds them are sometimes also not exposed to a sign language simply because they do not participate in a deaf community or attend a school where sign language is used. Despite their lack of access to any conventional language, these deaf individuals communicate with the hearing members of their community using gestures, called homesigns. Homesigns contain many, although not all, of the properties found in natural language (Goldin-Meadow, 2003). In some cases where access to sign language is the exception rather than the rule, a homesign system may continue to be used as a primary means of communication into adulthood (Coppola \& Newport, 2005).
\end{abstract}

\footnotetext{
(c) 2013 Elsevier Inc. All rights reserved.

Corresponding author: Elizabet Spaepen, Department of Psychology, University of Chicago, 5848 S. University Ave., Chicago, IL 60637. Phone: 773.834.1447 Fax: 773.702.0886, liesje@uchicago.edu.

'Permanent address: University of Connecticut, Department of Psychology, 406 Babbidge Road, Unit 1020, Storrs, CT 06269-1020, marie.coppola@uconn.edu

Publisher's Disclaimer: This is a PDF file of an unedited manuscript that has been accepted for publication. As a service to our customers we are providing this early version of the manuscript. The manuscript will undergo copyediting, typesetting, and review of the resulting proof before it is published in its final citable form. Please note that during the production process errors may be discovered which could affect the content, and all legal disclaimers that apply to the journal pertain.
} 
Studying adult homesign systems allows us to examine the impact that a language model can have on the status of different types of words in short-term memory-an issue that cannot be addressed in typically developing populations, who learn language from conventional language models. Here we ask whether words in a language system developed without conventional linguistic input serve as units in short-term memory, and whether words for numbers work in the same way as words for objects, actions, or attributes.

Homesigners use their gestures to communicate not only about objects, actions, or attributes, but also about number (Spaepen et al., 2011). The number gestures produced by adult homesigners, which are based on the gestures hearing individuals use to communicate about number (e.g., holding up 4 fingers to indicate four objects), are fully integrated into their homesign gesture sentences (e.g., four fingers held in the air, followed by a gesture for sheep, and then a gesture for $g o$, form a sentence describing four sheep leaving a pen), just as number words are integrated into spoken sentences and can either modify (e.g., "four sheep go") or stand in for ("four go") nouns (Coppola et al., , Spaepen \& Goldin-Meadow, in press). In this sense, homesigners' number gestures behave like words.

But the gestures homesigners use to represent number do not always behave like words. Homesigners convey the exact number of items in their gestures when describing small sets (e.g., they hold up 2 fingers to represent two items), but they convey an approximate number of items in their gestures when describing sets larger than 4 (e.g., they may hold up either 5 , 6 , or 7 fingers to represent six items). Homesigners display the same pattern in noncommunicative tasks; that is, when asked to match a target set of items, they do so exactly for small target sets (1-4) but only approximately for larger sets (>4). Homesigners thus do not have fully developed gestural or non-communicative representations of large exact number (Spaepen et al., 2011). Although the gestures homesigners use to communicate about large sets are not exactly correct, these gestures could have another property associated with words: they could function as single units in short-term memory, as do both conventional number words and conventional quantifiers that refer to approximate numerical values (like "few" and "many"). We ask here whether homesigners' number gestures function like words in this sense.

Human short-term memory capacity is limited: we are able to remember a list containing a small number of items better than a list containing a larger number of items (e.g. Miller, 1956; Cowan, 2001). Holding the number of items in a list constant, the capacity of shortterm verbal memory depends on phonological, but not semantic, properties of the words: it is harder to remember a list of words with more vs. fewer syllables_-seven vs. six (e.g. Baddeley et al., 1975) — but not words for bigger vs. smaller numbers-ten vs. six. In other words, spoken number words are summary symbols: symbols that represent the cardinal value of an entire set of items (e.g., a set of 8 sticks), and not the individual items within the set (i.e., not stick-stick-stick-stick-stick-stick-stick-stick). Remembering the number 8 should therefore be no harder than remembering the number 3 , and no harder than remembering any other one-syllable non-number word.

The same pattern holds for sign languages (Bavelier et al., 2008; Boutla et al., 2004; Hanson 1982, 1990; Wilson \& Emmorey, 2006), with the exception that signers have smaller digit or letter spans than speakers when required to recall items in the exact order in which they were presented, presumably because of the nature of echoic (as opposed to visual) memory. When tested using free recall of a list of words (rather than ordered recall of the list), signers and speakers, both native bilinguals in English and American Sign Language (ASL) and monolinguals of each language, perform comparably (Hanson 1982, 1990; Boutla et al., 2004; Bavelier et al., 2008). Importantly, holding the number of items in a list constant, the short-term memory capacity for signs depends on form, not meaning (Wilson \& Emmorey, 
1998) ${ }^{1}$ - longer signs (i.e., signs that traverse relatively long distances, a form distinction) are harder to recall than shorter signs (i.e., signs that do not change in location), an effect analogous to the length effect found in spoken language (longer words are harder to recall than shorter words). Thus, signers, like speakers, treat their words for numbers as summaries for the quantities they represent, making the sign for the number 8 no harder to remember than the sign for the number 3 .

The gestures homesigners use to represent number could function as summaries of the cardinal value of sets (summary symbols), but they could also function as representations of individual items within a set: each finger raised could represent another object in the set. In this case, a homesigner's gesture for " 8 " sticks would be better described as stick-stickstick-stick-stick-stick-stick-stick and should therefore take up more short-term memory resources during recall than a gesture for "3" sticks (i.e. stick-stick-stick). Do homesigners' number gestures behave as summary symbols for sets, or as representations of individual items within a set in short-term memory?

To address this question, we compare homesigners' recall of number gestures with their recall of gestures for objects, actions, and attributes, gestures whose forms map as wholes onto their referents (e.g., the gesture for child, a one-handed gesture produced with the knuckles of the non-thumb fingers flexed at the second joint, palm facing away from the body, with short repeated downward movements). We therefore gave homesigners two short-term memory tests, one containing gestures referring to numbers (digits), and one containing gestures referring to objects, actions, or attributes (nouns, verb, adjectives). To control for the impact that the manual modality might have on short-term memory, we also tested five deaf individuals fluent in Nicaraguan Sign Language (NSL) on digit span and noun/verb/adjective span tests in NSL. In addition, to control for the impact that schooling might have on short-term memory (the homesigners were all unschooled), we tested nine hearing Spanish speakers in Nicaragua who had not attended school on both tests in Spanish. Although it might have been preferable to control for communication modality and schooling within the same population, it is nearly impossible to find unschooled signers in Nicaragua simply because almost all signers learn NSL at school. We therefore controlled for these factors using separate populations.

If the homesigners' recall of gestures for objects, actions, and attributes resembles the patterns found for spoken words (in hearing speakers) and signs (in NSL signers), we will have evidence, the first of its kind, that lexical items can develop without linguistic input and can function as units in short-term memory. These findings can then serve as a backdrop against which to evaluate the status of homesigners' number gestures. Number gestures could map as wholes onto their referents (serving as summaries of cardinal values for sets and as single units in short-term memory), or could instead map onto the parts of their referents (representing each individual item within a set and serving as multiple units in short-term memory). Our findings thus promise to shed light on the nature of the numerical devices that can develop among adults who live in a numerate culture but lack a linguistic model.

\footnotetext{
${ }^{1}$ It is not known whether number of selected fingers, an aspect of phonological complexity in sign, affects short-term memory; in contrast to movement length in sign (or number of syllables in speech), this type of complexity does not increase the overall length of a word and thus might not have an impact on short-term recall.
} 


\section{Method}

\section{Participants}

We tested four homesigners in Nicaragua, ages 22-32 (3 men), who did not interact with one another. The homesigners' hearing losses prevented them from acquiring Spanish and they did not have access to Nicaraguan Sign Language (NSL). All four used only homesign to communicate with the hearing individuals around them. We also tested five signers, ages 21-27 (1 male), who were all early learners of NSL (starting before age 6 when they entered the Melania Morales Center for Special Education in Managua). The signers had an average of 15.4 years of NSL exposure in school, and used NSL exclusively to communicate. Finally, we tested nine hearing Spanish speakers in Nicaragua, ages 16-40 (3 males), who had never had formal schooling.

\section{Tasks presented to the homesigners}

Each homesigner was given two versions of the span recall task: digit span and noun/verb/ adjective span. In both versions, homesigners watched videos of actors producing 2-, 3-, and 4-unit sequences of number or non-number gestures. After each sequence, they were asked to repeat the sequence.

Digit Span-Gestures used in this task were those spontaneously produced by each of the homesigners to communicate about number. To test for the impact that size of number might have on recall, we divided the trial types into three types: (1) small number sequences contained gestures for 2 (index and middle finger) and 3 (index, middle, and ring fingers); (2) medium number sequences contained gestures for 4 (four fingers on one hand) and 5 (four fingers and thumb); (3) large number sequences contained gestures for 8 (index, middle, and ring fingers of one hand plus all fingers and thumb of the other hand) and 9 (four fingers of one hand plus all fingers and thumb of the other hand). To ensure that the homesigners did not simply ignore the " 5 " hand in the large number trials, the actor switched the hand that represented the 5 for numbers within a trial. ${ }^{2}$ Each sequence contained only two gesture forms; thus a 2-digit medium trial might be the sequence $4-5$ or the sequence 5-5; a 3-digit medium trial might be 4-5-4, 5-4-5, 4-4-5, etc. Homesigners did not have to use the same finger configurations as the model for their response to be counted as correct (e.g., they could use the thumb, index, and middle fingers for 3). All three trial types were intermixed with a given span length $(2,3$, or 4 digits), and were presented in a fixed, pseudo-random order.

Noun/Verb/Adjective Span-This task was identical to the digit span task, except that participants saw an actor produce "gestural emblems" (gestures that are codified within a culture-see Ekman \& Friesen, 1969); these gestures are commonly used by hearing speakers in Nicaragua (e.g., the "child" gesture described earlier) and all had been fully incorporated into each homesigner's gesture system, as well as into NSL. As in the digit span task, each sequence contained two gesture types; thus a 2-unit sequence might be childcome or come-come; a 3-unit sequence might be come-child-come, child-come-child, comecome-child, etc. Visually similar gestures were paired with one another (e.g., "child" and "come," come is produced with one hand, palm facing down, with the knuckles of the nonthumb fingers repeatedly flexing at the first joint). Pairs of noun/verb/adjective gestures were chosen to be as comparable as possible to the pairs of number gestures (e.g., $t w o$ vs. three) in terms of perceptual similarity. In addition, gestures were equated for number of

\footnotetext{
${ }^{2}$ Unlike conventional sign languages where switching the dominant hand across presentations of the same number would be unusual and therefore distracting for signers watching the actor, homesigners are not consistent in the hand they use for number gestures; switching dominant hands was therefore not inconsistent with the homesigners' own gestures for these quantities.
} 
hands; thus, two noun/verb/adjective sequences contained one-handed gestures (to be comparable to the small and medium trial types in the digit span task) and one sequence contained two-handed gestures (to be comparable to the large trial type in the digit span task). ${ }^{3}$

\section{Tasks presented to the NSL signers}

The stimuli presented to the NSL signers in the digit span task were identical to those presented to the homesigners for the small (2-3) and medium (4-5) number trials, but not for the large (8-9) number trials; signs for 8 and 9 are both made with one hand, rather than two, in NSL. The same one-handed stimuli presented to the homesigners in the noun/verb/ adjective task were presented to the signers (these Nicaraguan gestures have all been incorporated into NSL as lexical items). The two-handed stimuli shown to the homesigners were replaced by one-handed stimuli for the NSL signers, to be comparable to their digit span trials, which were all one-handed.

Signed digits are not ideal stimuli for span recall because the perceptual similarity of the digits often makes number signs difficult to distinguish from one another (Wilson \& Emmorey, 2006). Note, however, that this concern applies to both the homesigners and the signers in our study. For two of the trial types (small and medium), the stimuli we presented to the NSL signers and the homesigners were identical. For the large trial type, perceptual similarity between stimuli presented to the two groups was comparable (i.e., 9 contains one more finger than 8 in both homesign and NSL, see the Appendix), as was stimulus complexity (i.e., homesigners' gestures for large numerical trials require two hands, but NSL signs for 8 and 9 include movement and a complex handshape [Brentari, 1998], making them comparably complex). As a result, any differences we find between groups on this task are not likely to be due to differences in perceptual similarity or complexity between the stimuli.

\section{Tasks presented to the Spanish speakers}

The hearing speakers saw and heard Spanish number and non-number words spoken by a native Spanish speaker presented in video clips on a computer. The hearing speakers' videos were identical to the signers' and homesigners' videos except that a different actor, a native Spanish speaker, spoke the digit strings, rather than gesturing or signing them. Just as the gestures and signs were equated across tasks for number of hands, the Spanish speakers' targets were equated for number of syllables across tasks. Thus, the hearing controls heard one pair of one-syllable words (to be comparable to their small number trials in the digit span task: dos-tres) and two pairs of two-syllable words (to be comparable to their medium and large number trials). See the Appendix for a list of the stimulus trial types presented to all three groups for both tasks.

\section{Procedure}

Participants watched a video clip of an actor gesturing, signing, or speaking the items and then were instructed to repeat the string. If participants asked to re-watch a trial before responding, the experimenter replayed the clip. If the participant completed an attempt to repeat the string and then asked to retry the trial, both answers were recorded, but only the first attempt was used in the analyses presented here.

\footnotetext{
${ }^{3}$ The two gestures in one of the one-handed pairs in the noun/verb/adjective task turned out to be very difficult to distinguish from one another because of the way the stimuli were produced on videotape. As a result, the data from these trials were excluded from the analyses. See the Appendix for more information.
} 
Presentation of the noun/verb/adjective span task and the digit span task was counterbalanced across participants within each of the three groups. In both tasks, homesigners saw six 2-unit trials, twelve 3-unit trials, and six 4-unit trials. The two control groups saw six 2-unit trials, twelve 3-unit trials, twelve 4-unit trials, and six 5-unit trials to avoid the possibility of ceiling effects.

Trials were presented in increasing difficulty. That is, participants completed all of the 2unit span trials ( 6 trials per participant) before proceeding to the 3 -unit span trials ( 12 per participant), after which they saw the 4-digit trials (6 or 12 per participant, depending on participant group), and the NSL signers and Spanish speakers then saw all of the 5-digit trials (6 per participant). If participants started to answer after seeing the first two elements of the string, they were stopped and told to wait until the end of a trial before answering. They then re-watched the entire string.

All responses were videotaped and coded for accuracy by one coder blind to the hypotheses of the study. A second coder, also blind to the hypotheses of the study, coded 13 of the 18 participants (all 4 of the homesigners, all 5 of the signers, and 4 of the 9 speakers). Intercoder reliability across all groups was $94 \%$ for the digit span task and $94 \%$ for the word span task. In all cases, discrepancies were notation errors and were easily resolved by re-watching the trial.

\section{Results}

Figure 1 presents the proportion of correctly remembered sequences on the digit span and noun/verb/adjective span tasks for the (a) homesigners, (b) NSL signers, and (c) unschooled Spanish speakers. Because requiring that items be recalled in the order in which they were presented reduces the recall of visually-presented words relative to aurally-presented words (Bavelier et al., 2008), sequences did not have to be recalled in the order presented in the stimulus to be counted as correct (e.g., if 2-3-2 was the target sequence, 2-2-3 was also coded as correct; however, 2-3-3 was not). If order is included as a criterion, the patterns do not change.

We compared the noun/verb/adjective span and the digit span tasks across the three participant groups, using a $2 \times 3 \times 3$ repeated measures ANOVA (task $\times$ participant group $\times$ span length) on the arcsine transformed data ${ }^{4}$ and found a significant interaction between task and participant group, $\mathrm{F}(2,15)=10.35$, $\mathrm{p}<0.01$, main effects of span length $(F(2,15)=37.30, p<0.001)$ and participant group $(F(2,15)=5.19, p<0.05)$, and no interaction between span length and participant group $(\mathrm{F}(2,15)=1.03, \mathrm{p}=0.38)$. A Tukey post-hoc analysis revealed that the homesigners differed significantly from the signers $(p<0.05)$ and marginally from the speakers $(\mathrm{p}=0.09)$, but the two control groups did not differ from one another $(\mathrm{p}=0.37)$.

We also analyzed the digit span and the noun/verb/adjective tasks separately, collapsed across span lengths, for the three participant groups, using one-way ANOVAs. These analyses revealed an effect of participant group in the digit span task $(\mathrm{F}(2,15)=8.53$, $\mathrm{p}<0.01)$, but not in the noun/verb/adjective task $(\mathrm{F}(2,15)=1.44, \mathrm{p}=0.27)$. A Tukey post-hoc analysis on the digit span task showed that homesigners differed significantly from the deaf signers $(\mathrm{p}<0.01)$ and from the hearing speakers $(\mathrm{p}<0.05)$. The two control groups did not differ from one another $(\mathrm{p}=0.40)$. In other words, homesigners performed worse than the two control groups on the digit span task, but the three groups did not differ in their

\footnotetext{
${ }^{4}$ All analyses presented were performed on arcsine-transformed data. Although this transformation helps resolve some of the issues with proportions, we recognize that it is an imperfect solution.
} 
performance on the noun/verb/adjective task, indicating that the type of item to be remembered (numbers vs. non-numbers) affected recall in the homesigners but not in the signers or Spanish speakers. 5

Several findings are important to note in these data. First, the NSL signers' excellent recall of both number and non-number signs suggests that both types of signs can function as units in short-term memory. Given that NSL is a conventional language with a very short history (Senghas \& Coppola, 2001), finding recall patterns that resemble patterns found in more established sign languages (e.g., Boutla et al., 2004; Bavelier et al., 2008) is itself a noteworthy result.

Second, the fact that homesigners' recall of gestures for nouns, verbs, and adjectives was no different from the recall of comparable signs in NSL signers and words in Spanish speakers suggests that at least some types of homesigns do function as units within short-term memory. In particular, the lexical items in homesign systems that denoted kinds of objects, actions, or attributes achieved word status in short-term memory, despite the fact that they were not learned in the context of a conventionalized natural language.

Third, the homesigners' poor recall of gestures for numbers, compared both to their superior recall of non-number gestures and to the signers' and speakers' recall of both number and non-number signs/words, suggests that number gestures do not function as units in shortterm memory for homesigners. For homesigners, number gestures do not appear to serve as summaries of cardinal values of sets, as they do for speakers of new or established conventional languages.

If the homesigners' number gestures are not serving as summaries of entire sets, we might expect that each additional finger in the target stimulus would add to the homesigners' recall burden. We would then predict that recall of sequences containing large numbers ( 8 and 9 ) would be worse than recall of sequences containing medium numbers ( 4 and 5 ), which, in turn, would be worse than recall of sequences containing small numbers ( 2 and 3 ).

To explore this possibility, we compared the three groups on the effect of numerical size in the digit span task (see Figure 2). To avoid the possibility of ceiling effects that might arise as a function of the control groups' perfect performance on some span lengths, we equated for performance by subjecting to statistical analysis only the data from spans showing significant errors: 2- and 3-digit spans for homesigners (63\% accuracy overall, $77 \%$ when large number trials were removed); 4- and 5-digit spans for NSL signers (84\% accuracy overall), and 3-, 4- and 5-digit spans for Spanish speakers (80\% accuracy overall).

Collapsing over span size, we conducted a $3 \times 3$ repeated measures ANOVA (trial type: small, medium, large) $\times$ participant group: homesigners, signers, speakers) and found an interaction between trial type and participant group, $\mathrm{F}(2,15)=5.69, \mathrm{p}=0.01$. To explore this interaction, we conducted $2 \times 3$ repeated measures ANOVAs (participant group $\times$ trial type) on each pair of participant groups. We found that homesigners were marginally more affected by numerical size (i.e., trial type) than the NSL signers, $F(1,7)=4.97, \mathrm{p}=0.06$, and significantly more affected by numerical size than the Spanish speakers, $F(1,11)=10.12$, $\mathrm{p}<0.01$; the two control groups did not differ with respect to the impact that numerical size

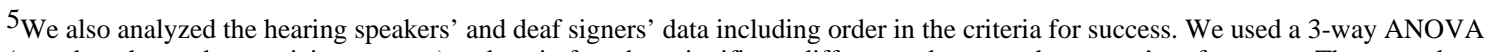
(span length $\times$ task $\times$ participant group) and again found no significant differences between the groups' performance. The control groups' comparable performance may seem surprising given data suggesting smaller spans for signed vs. spoken languages. However, schooling (and literacy) have also been found to affect span length (see Ostrosky-Solís \& Lozano, 2006; Reis et al., 2003). Indeed, when we include order as a criterion for success, we not only find differences between signers vs. speakers (i.e., across modalities), but we also find differences between schooled vs. unschooled speakers (i.e., across levels of schooling, see Scribner \& Cole, 1981). These two factors, modality and schooling, may have balanced each other out, resulting in similar patterns of performance in our control groups.
} 
had on their performance, $F(1,12)=0.05, p=0.82$. Looking at Figure 2, we see that homesigners performed better on both the small $(2,3)$ and medium $(4,5)$ number trials than on the large $(8,9)$ number trials for 2-digit spans. When asked to remember more digits (i.e., the 3-digit spans), homesigners exhibited the stepwise function one might expect to see if additional fingers are adding cumulatively to their recall burden: as the number of fingers in the individual gestures within a sequence increased, homesigners' accuracy decreased from small $(2,3)$, to medium $(4,5)$, to large $(8,9)$ number trials.

There is a possible confound in these data: The target stimuli for large numbers $(8,9)$ consisted of two hands for the homesigners but only one hand for the signers. The homesigners' greater difficulty on large-number trials might therefore stem from the fact that they had to remember two hands on these trials, but only one hand on the small and medium number trials. Note that this explanation will not account for the homesigners' worse performance on medium number trials than on small number trials in the 3-digit spans, both of which involve a single hand, see Figure 2a, right-hand graph). Nevertheless, the mere presence of a second hand might have made the large number trials difficult to recall for the homesigners, rather than the fact that the second hand in these gestures represented additional items in the set.

To test this possibility, we compared the homesigners' recall of one- vs. two-handed gestures in the digit span task to their recall of one- vs. two-handed gestures in the noun/ verb/adjective span task. The second hand in noun/verb/adjectives gestures does not convey additional information but instead forms a single whole with the first hand and thus should not put an additional burden on memory (e.g., "relative," produced by tracing a line with the index finger of one hand from the wrist to elbow along the inside of the forearm of the other arm). Thus, in contrast to two-handed number gestures, two-handed noun/verb/adjective gestures should be no harder to recall than one-handed noun/verb/adjective gestures (e.g., "child").

Figure 3 presents the proportion of sequences the homesigners recalled correctly in the (a) digit span task and (b) noun/verb/adjective span task as a function of the number of hands, one vs. two, in the target stimulus (only the 2- and 3-unit trials are included in the figure because the homesigners' recall on the 4-digit spans was so low that we were concerned about floor effects). We conducted a repeated measures ANOVA and found a significant interaction between number of hands and task, $\mathrm{F}(1,3)=10.59, \mathrm{p}<0.05$. As predicted, on both 2-digit and 3-digit spans, homesigners recalled significantly more sequences made with one hand than with two hands for number gestures, but not for noun/verb/adjective gestures. These results suggest that the difficulty homesigners had with the large numbers on the digit span task was not due simply to the fact that they had to track two hands, as they did not have the same difficulty with two-handed targets on the noun/verb/adjective span task. We suggest that the second hand in the homesigner's number gestures represented for them additional items in the set, which made recall more difficult.

An error analysis revealed that homesigners also made a different type of error in the large number trials in the digit span task, compared to small and medium number trials and compared to any of the trials in the noun/verb/adjective task-intrusion errors, that is, producing digits during recall that were not presented anywhere in the task (i.e., 6, 7 or 10); $34 \%$ of the homesigners' trials in the large number condition contained intrusions, compared to $0 \%$ of their small and medium number trials, and $0 \%$ of their trials in the noun/verb/ adjective task. Neither the hearing controls nor the NSL signers produced any intrusions on any type of trial in the digit span task. The hearing controls did produce intrusions in the noun/verb/adjective span task: 3 of the 9 participants produced at least one word during recall that had never been presented anywhere in the task (saying "paz" instead of the target 
word "mas" or "soy" instead of the target word "sol"). These three participants produced a total of 16 intrusions, or $15 \%$ of the one-syllable trials presented to the speaking participants overall. The homesigners never produced intrusions in the word span task, and the NSL signers produced no intrusions on either task. Thus, homesigners showed both a higher rate and a different pattern of intrusions than either of the comparison groups.

\section{Discussion}

Our findings provide the first evidence that the gestures homesigners use to designate objects, actions, and attributes (Goldin-Meadow et al., 1994) function as units in short-term memory, just words and signs do in conventional spoken and sign languages. The findings thus add weight to the claim that homesign functions in many respects like a natural language.

Importantly, however, this parallel does not extend to homesigners' gestures for numbers. Number gestures in homesign do not seem to have the full representational power of count words in conventional language, signed or spoken, nor the power of other gestures in the homesigners' own vocabularies. Rather, the homesigners' gestures for number are better described as indexes for individual members of a set, chunked first into units of five (hands) and then into units of one (fingers). Homesigners are not likely to be analyzing the number gestures in terms of individual fingers, else they would not have been able to recall as many sequences of large numbers as they did (the number of fingers in these trials, if each were held independently in memory, would exceed the typical short-term memory span, Boutla et al., 2004). We suspect that the homesigners were encoding the number gestures as some combination of hands and fingers; for example, 9 would not be encoded as a series of "ones," but rather as "group(hand)+one-one-one-one." This strategy could explain why homesigners' recall of the medium numbers $(4,5)$ is perfect on the 2-digit span-medium trial types ought to be easy to recall if homesigners encode 5 as "one chunked group" rather than "one-one-one-one-one."

Note that we are not arguing that the homesigners have a summary symbol for "five" or even "hand," as the data do not support this claim. In particular, one of the numbers homesigners produced as an intrusion error during the task was "10," even though that number never appeared on the memory span test. If they stably encoded 10 as "two hands," then such an error should not have occurred.

Rather, we argue that homesigners use the fact that hands chunk fingers into sets of five to their advantage, just as chunking can increase the number of objects infants and adults can track using their object tracking system (Feigenson \& Halberda, 2004; Halberda et al., 2006; Feigenson \& Halberda, 2008). This strategy can explain why we see only a marginal effect of numerical size in homesigners' performance on the digit span task, particularly in the medium range, since "five" may be a privileged and easily chunked handshape even though it does not function as a stable summary symbol. In contrast, we do see an effect of adding another hand to the to-be-remembered number, but only when homesigners need to remember digits, not when they need to remember other words in their homesign systems (see Figure 3).

The data also suggest that homesigners used their pre-linguistic small exact number system, or parallel individuation (see Carey, 2009 for a review), to encode gestures where possible. Their equally good performance for small numbers ( 2 and 3 ) on 2- and 3-digit trials, but not for larger numbers (4 and 5 or 8 and 9), likely reflects the fact that homesigners were able to use their small exact number system to assess the small sets quickly and accurately, which improved recall. 
The homesigners' number gestures do not function as single words in short-term memory in that they do not reduce the short-term memory load posed by the enumerated items as consistently and effectively as do the number words in conventional languages. But homesigners' number gestures do function as words in the sense that they are incorporated into gesture sentences and serve to modify (e.g., 7 birds drink) and stand in for (e.g., 7 drink) nouns in those sentences (Coppola et al., in press), suggesting that these two functions need not go hand-in-hand.

The fact that the homesigners do not encode number gestures as single chunks may have consequences for their ability to compute and represent large exact numbers, even in noncommunicative contexts (see Spaepen et al., 2011). However, we do not yet have evidence for a direct link between the number gestures homesigners use to communicate and the representations of number that they use in non-communicative situations. In addition, we do not yet understand how these two representational capacities develop. Future research is needed to explore whether teaching young homesigners summary symbols for large exact numbers can have an impact on their non-communicative representations of number, and vice versa.

Our findings are particularly striking because homesigners have almost certainly seen hearing individuals in their culture using the gestures shown in both the digit span and noun/ verb/adjective span tasks. The gestures in the noun/verb/adjective span task are gestural emblems commonly used by hearing Spanish speakers in Nicaragua (Coppola, 2007), and the gestures used in the digit span task (holding up fingers to represent the number being communicated) are the most common way hearing people gesture about number. The oneto-one correspondence that can be established easily between fingers and countable objects facilitates communication about number for homesigners, but may lead them down a garden path - they may fail to see that two fingers held in the air can stand for a set of 2 items. As we have shown here, this failure can limit the cognitive benefit that homesigners' number gestures convey.

Previous research on homesigners suggests that linguistic input, in the form of a count list, may be vital to developing concepts of large exact numbers (larger than 3 or 4 , Spaepen et al., 2011). Moreover, other cultures whose languages do not have conventionalized count systems or words for numbers higher than 4 or 5 also show deficits when performing tasks that require conceptual representations of large exact numbers (Gordon, 2004; Pica, et al., 2004). Without such conceptual representations, the gestures homesigners use to represent number would not be predicted to function like single units in short-term memory. Our findings confirm this prediction.

It is important to point out that gestures that look like indexes of individual items can function like summaries of sets if they have been learned as part of a counting system. The signs for 1 through 5 in NSL (and other conventional sign languages such as American Sign Language) resemble the tallies used by the homesigners (i.e., extending 1, 2, 3, 4, or 5 fingers). But the NSL signers in our study recalled the same number of sequences with small $(2,3)$ and medium $(4,5)$ numbers as they did with large $(8,9)$ numbers (whose forms are one-handed and arbitrary, e.g., " 8 " in NSL consists of a hand configuration with the thumb, index and middle fingers extended, palm facing the body or the signer's midline, with an outward wrist rotation), even in the 4- and 5-digit spans where their recall was not perfect (see Figure 2b). When learned in the context of a counting system, gestures that look like tallies can take on an arbitrariness that allows them to function as summaries for sets.

Our study suggests that numerical gestures developed without benefit of conventional linguistic input do not function like single units in short-term memory. However, the study 
has certain limitations that require further research. First, although the stimuli were designed to minimize differences in perceptual similarity, we cannot rule out the possibility that the homesigners' gestures for 8 and 9 were particularly difficult to distinguish from one another. We are currently addressing this issue by studying earlier cohorts of Nicaraguan signers, who use two hands to represent 8 and 9, but for whom the signs are presumably summaries of cardinal values. We would expect these signers to process signs for 8 and 9 no differently than they process signs for 4 and 5 despite the fact that it may be harder to distinguish between the signs for 8 and 9 than to distinguish between the signs for 4 and 5 .

Second, it is possible that the patterns we find in the homesigners' number gestures stem from their lack of experience with large (as opposed to small) numbers. Note, however, that the homesigners did recall sets containing gestures for 2 and 3 better than sets containing gestures for 4 and 5 (see Figure 2, 3-digit span length) even though all of these numbers are typically considered small. Moreover, the homesigners have jobs and participate within their communities and thus are likely to have had comparable exposure to larger numbers as the unschooled hearing adults.

Third, the homesigners have limited and less automatic access to Arabic numerals: they generally know Arabic numerals up to 5 but have idiosyncratic, limited knowledge beyond that. In this respect, they differ from NSL signers, who show robust knowledge of Arabic numerals (Flaherty \& Senghas, 2011). Although the facility of our uneducated hearing speakers with Arabic numerals was not tested, it is likely that they too can identify Arabic numerals well, and that this difference contributed to the homesigners' poorer performance on the digit span task. The two control groups may thus may have had multiple codes for numbers, but only one code for items on the noun, verb and adjective task (the Spanish speakers were illiterate and thus had limited access to written forms for these items, as did the NSL signers who had little knowledge of written Spanish). However, this possibility requires further research to link each participant's facility with Arabic numerals and written Spanish words with their performance on each task.

In sum, we have shown that homesigners have gestures that function in short-term memory just as the words and signs of a conventional language do. Natural language input is not necessary to develop words that behave as single linguistic units. However, the same status does not extend to number gestures. Homesigners do not have gestures for numbers that function as single units in short-term memory. In previous research (Spaepen et al., 2011), we showed that homesigners' gestures for number also do not have the representational power of the exact number words in a conventional language, although they do combine with other gestures in some contexts (Coppola et al., in press). Natural language input therefore appears to be necessary to develop a full system of words for exact numbers.

\section{Acknowledgments}

Supported by R01DC00491 from NIH/NIDCD to SGM, an NSF REESE Foundation grant to E. Spelke (DRL-0633955), P30 DC010751 from NIH to MC and Diane Lillo-Martin, and SBE-0541957 from NSF (Spatial Intelligence and Learning Center; Goldin-Meadow is a co-PI). We thank L. Applebaum, S. Carvajal-Raga, A. Guinan, and S. Namboodiripad for assistance in making stimuli, E. Carrigan, J. Fanghella, and J. Henner for assistance during data collection, and J. Goldsmith-Pinkham, F. Martin and K. Stangl for coding assistance. Finally, we thank K. Bock and two anonymous reviewers for thoughtful comments on a prior version of this manuscript. 


\section{APPENDIX}

\section{Stimuli for Digit Span Task}

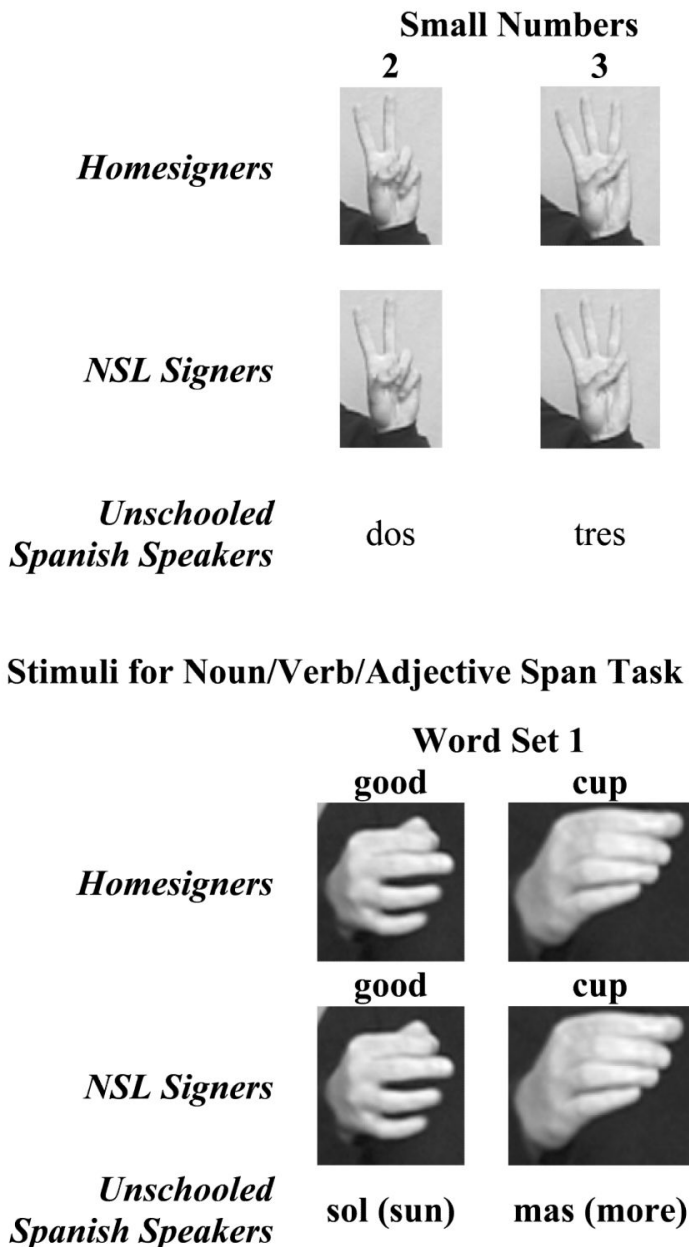

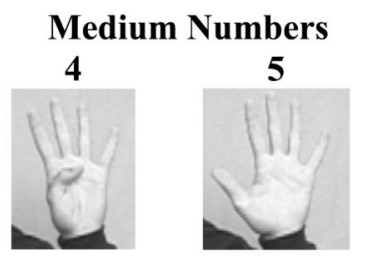
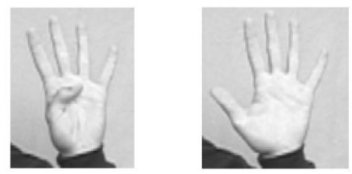

cuatro

cinco
Large Numbers

8
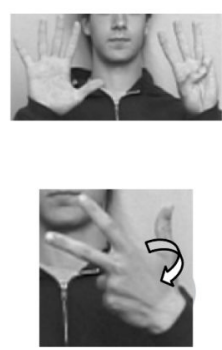

ocho
9
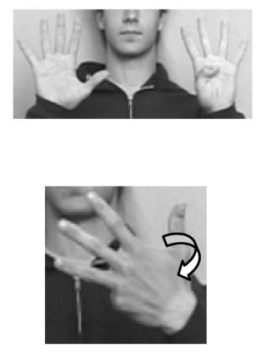

nueve
Word Set 2

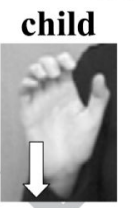

child

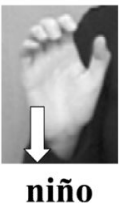

(child)

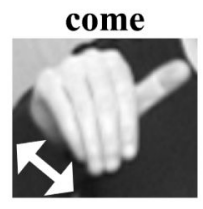

come

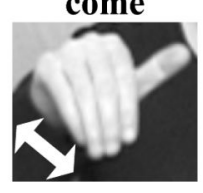

venir (come)

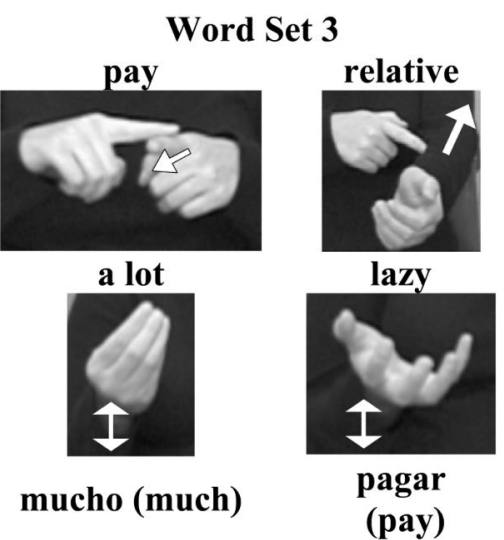

Figure A1. Still images of each of the sign or gesture pairs used in the digit span and noun/verb/ adjective span tasks

All videos were shown in color. The images presented for good and cup turned out to be too difficult to distinguish from one another when presented as stimuli in our experimental setup; as a result, the data from these pairs were not included in the analyses for either homesigners or signers.

\section{REFERENCES}

Baddeley A, Thomson N, Buchanan M. Word length and the structure of short-term memory. Journal of Verbal Learning and Verbal Behavior. 1975; 14:575-589.

Bavelier D, Newport E, Hall M, Supalla TS, Boutla M. Ordered short-term memory differs in signers and speakers: Implications for models of short-term memory. Cognition. 2008; 107(2):433-459. [PubMed: 18083155]

Boutla M, Supalla T, Newport L, Bavelier D. Short-term memory span: Insights from sign language. Nature Neuroscience. 2004; 7(9):1-6.

Brentari, D. A prosodic model of sign language phonology. MIT Press; Cambridge, MA: 1998.

Carey, S. The origin of concepts. Oxford University Press; USA: 2009. 
Coppola, M. Gestures to signs: The origins of words in Nicaraguan Sign Language; Paper presented at Current Issues in Sign Language Research; University of Köln, Köln, Germany. 2007;

Coppola M, Newport E. Grammatical Subjects in home sign: Abstract linguistic structure in adult primary gesture systems without linguistic input. Proceedings of the National Academy of Sciences. 2005; 102(52):19249-19253.

Coppola M, Spaepen E, Goldin-Meadow S. Communicating about quantity without a language model: Number devices in homesign grammar. Cognitive Psychology. in press.

Cowan N. The magical number 4 in short-term memory: a reconsideration of mental storage capacity. Behavioral and Brain Sciences. 2001; 24:87-185. [PubMed: 11515286]

Ekman P, Friesen W. The repertoire of nonverbal behavior categories, origins, usage, and coding. Semiotica. 1969; 1:49-98.

Feigenson L, Halberda J. Infants chunk object arrays into sets of individuals. Cognition. 2004; 91:173190. [PubMed: 14738772]

Feigenson L, Halberda J. Conceptual knowledge increases infants' memory capacity. Proceedings of the National Academy of Sciences of the United States of America. 2008; 105(29):9926-9930. [PubMed: 18626025]

Flaherty M, Senghas A. Numerosity and number signs in Deaf Nicaraguan adults. Cognition. 2011; 121(3):427-436. [PubMed: 21899832]

Goldin-Meadow, S. The resilience of language: What gesture creation in deaf children can tell us about how all children learn language. In: Werker, J.; Wellman, H., editors. Essays in Developmental Psychology Series. Psychology Press; New York: 2003.

Goldin-Meadow S, Butcher C, Mylander C, Dodge M. Nouns and verbs in a self-styled gesture system: What's in a name? Cognitive Psychology. 1994; 27:259-319. [PubMed: 7828423]

Gordon P. Numerical cognition without words: evidence from Amazonia. Science. 2004; 306:496499. [PubMed: 15319490]

Halberda J, Sires SF, Feigenson L. Multiple spatially-overlapping sets can be enumerated in parallel. Psychological Science. 2006; 17(7):572-576. [PubMed: 16866741]

Hanson V. Short-term recall by deaf signers of American Sign Language: Implications of encoding strategy for order recall. Journal of Experimental Psychology. Learning Memory and Cognition. 1982; 8(6):572-583.

Hanson V. Recall of order information by deaf signers: Phonetic coding in temporal order recall. Memory and Cognition. 1990; 18(6):604-610. [PubMed: 2266862]

Miller G. The magical number seven plus or minus two: some limits on our capacity for processing information. Psychological Review. 1956; 63(2):81-97. [PubMed: 13310704]

Ostrosky-Solís F, Lozano A. Digit span: Effect of education and culture. International Journal of Psychology. 2006; 41(5):333-341.

Pica P, Lemer C, Izard V, Dehaene S. Exact and approximate arithmetic in an Amazonian indigene group. Science. 2004; 306:499-503. [PubMed: 15486303]

Reis A, Guerreiro M, Petersson K. A sociodemographic and neuropsychological characterization of an illiterate population. Applied Neuropsychology. 2003; 10(4):191-204. [PubMed: 14690800]

Scribner S, Cole M. Unpackaging literacy. Writing: The nature, development, and teaching of written communication. 1981; 1:71-87.

Senghas A, Coppola M. Children creating language: How Nicaraguan Sign Language acquired a spatial grammar. Psychological Science. 2001; 12(4):323-328. [PubMed: 11476100]

Spaepen E, Coppola M, Spelke E, Carey S, Goldin-Meadow S. Number without a language model. Proceedings of the National Academy of Science of the United States of America. 2011; 108(8): 3163-3168.

Wilson M, Emmorey K. A “word length effect” for sign language: Further evidence on the role of language in structuring working memory. Memory \& Cognition. 1998; 26:584-590. [PubMed: 9610126]

Wilson M, Emmorey K. Comparing sign language and speech reveals a universal limit on short-term memory capacity. Psychological Science. 2006; 17:682-683. [PubMed: 16913950] 


\section{Highlights}

Homesigners produce number gestures though they lack a conventional language model.

Homesigners recall nouns, verbs \& adjectives, but not numbers, as well as signers.

Homesign number gestures may not summarize sets: recall worsens as value increases.

Homesign gestures for non-numbers act as words in memory, but number gestures do not.

Numbers may only serve as units in memory if learned within a conventional language 


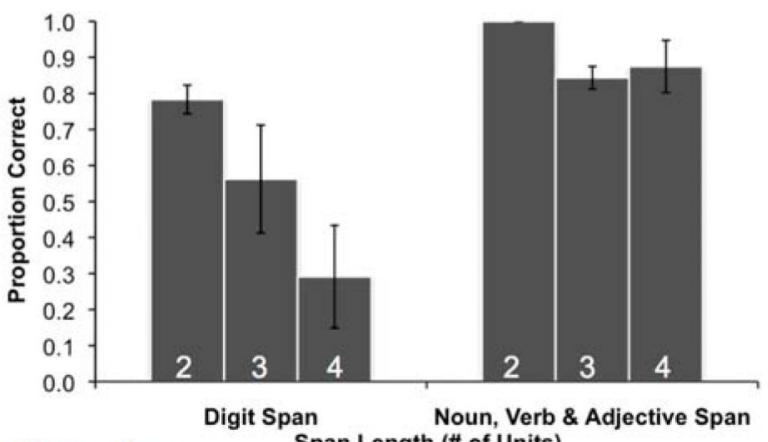

(a) Homesigners

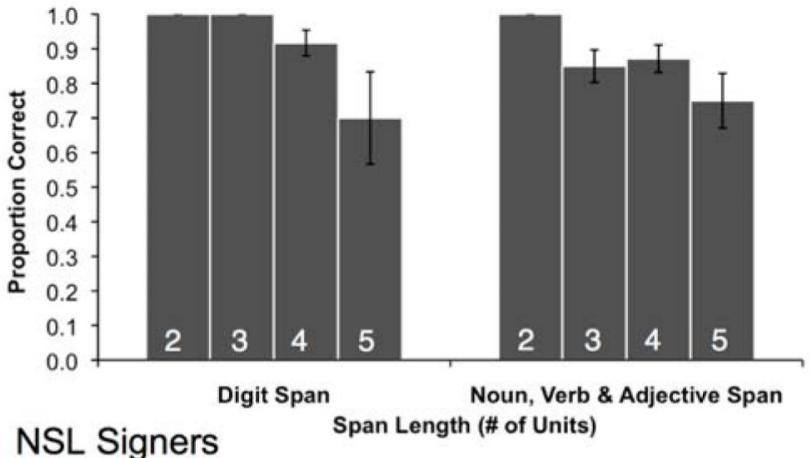

(b) NSL Signers

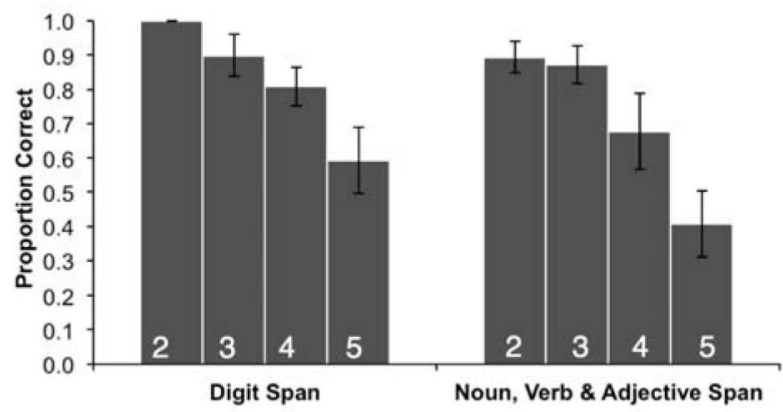

(c) Spanish
Speakers

Span Length (\# of Units)

Figure 1.

Proportion of correctly recalled sequences by (a) homesigners (b) NSL signers and (c) unschooled Spanish speakers on the Digit Span and the Noun/Verb/Adjective Span task as a function of the number of number of units in the target (i.e., span length). Error bars indicate standard error. 

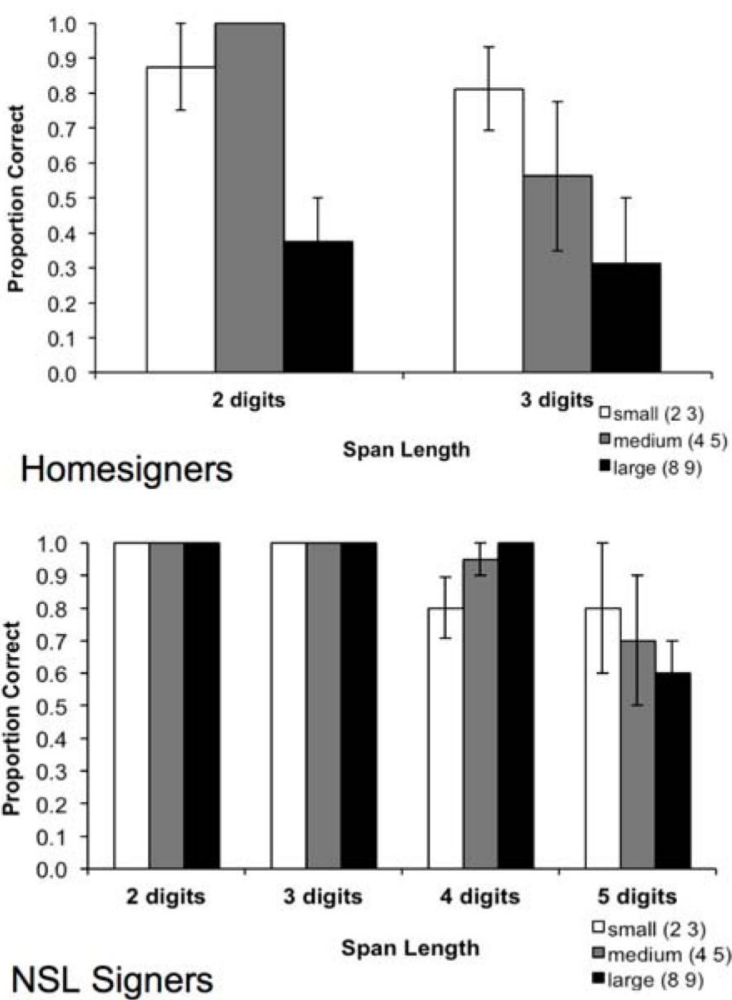

(b) NSL Signers

$$
\begin{aligned}
& \text {-medium (4 5) } \\
& \text { - large (8 9) }
\end{aligned}
$$

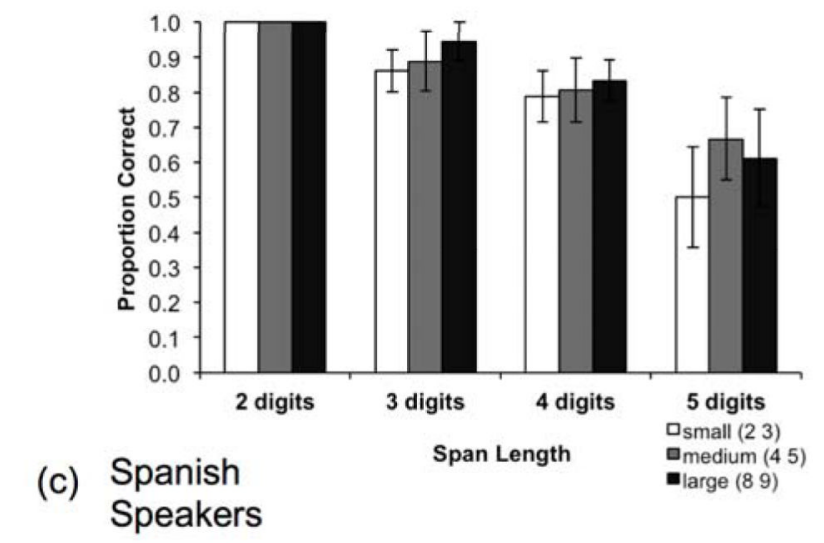

Figure 2.

Proportion of correctly recalled sequences by (a) homesigners (b) NSL signers and (c) unschooled Spanish speakers on the digit span task as a function of trial type. Trials were characterized by the size of the numbers they contained: small ( 2 and 3 only), medium ( 4 and 5 only), and large (8 and 9 only). Error bars indicate standard error. 


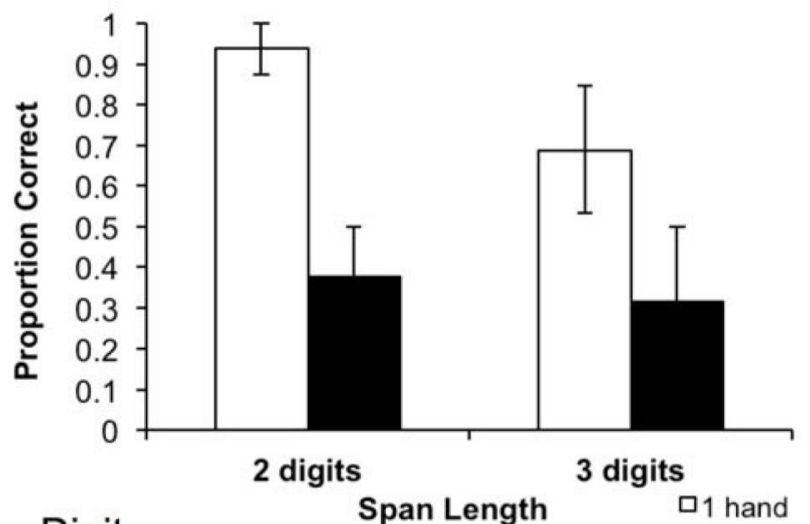

(a)
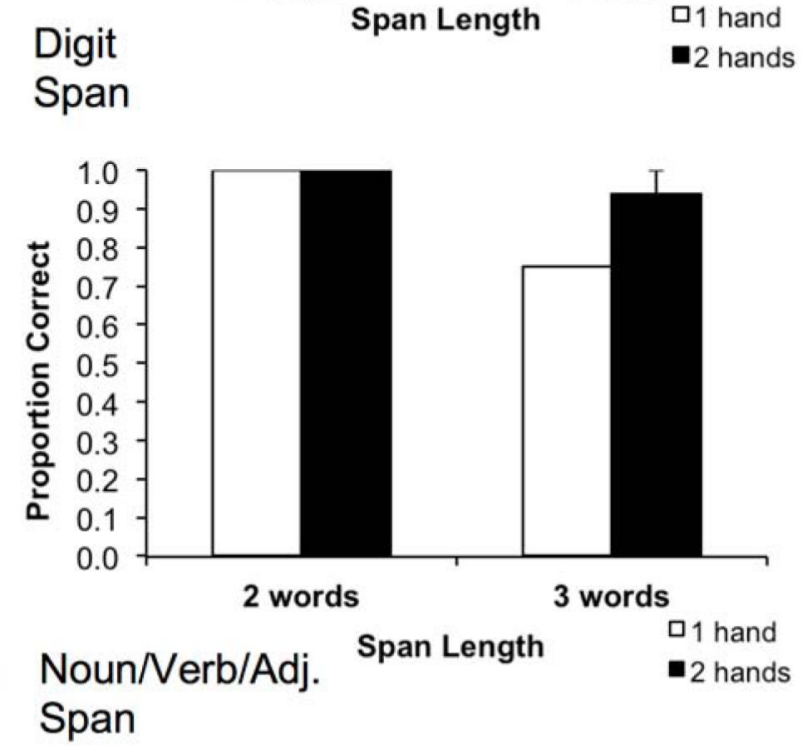

Figure 3.

Proportion of correctly recalled sequences in the homesigners on the (a) the Digit Span task and (b) the Noun, Verb \& Adjective span task as a function of number of hands (one vs. two) in the target stimulus. Error bars indicate standard error. 